\title{
Effect of Carrying Style on Posture Score in Adolescents with Musculoskeletal Pain
}

\author{
Nuanlaor Thawinchai* and Kannikar Funprom \\ Department of Physical Therapy, Faculty of Associated Medical Sciences, \\ Chiang Mai University, Chiang Mai 50200, Thailand \\ *Corresponding author.Email: nuanlaor.thawinchai@cmu.ac.th \\ https://doi.org/10.12982/CMUJNS.2019.0036
}

Received: January 30, 2019

Revised: March 6, 2019

Accepted: April 24, 2019

\begin{abstract}
Although the weight of bag is the primary risk factor for poor posture, the carrying style should be another risk factor. The Reedco's posture score (RPS) is an alternative tool in clinical setting to evaluate posture changes. The purpose of this study was to investigate posture in adolescents who had musculoskeletal pain with and without carrying backpacks using the RPS. This study was a single-factor design for repeated measures. The participants were assessed for their posture in three different conditions: carrying no backpacks, carrying backpacks with one strap, and carrying backpacks with two straps, using the RPS. The results showed a statistically significant decrease in the RPS between with and without backpacks $(P<0.001)$. In addition, the RPS with one strap had decreased statistically significant postural alignment score when compared to the RPS with two straps $(P=0.017)$. The prevention or intervention program should include not only the load of the backpack but also the carrying style.
\end{abstract}

Keywords: Symmetrical carrying, Asymmetrical carrying, Reedco's posture score 


\section{INTRODUCTION}

The rate of musculoskeletal (MS) pain in adolescents is found to be on an increasing trend in Thailand (Sihawong, et al., 2006; Chatchawan et al., 2013; Deesungnern and Thawinchai, 2014). Although schoolbag load is a major risk factor that causes MS pain and hospitalization (Chiang et al., 2006; Chow et al., 2007; Mooreet al., 2007; Negrini and Negrini, 2007; Sharanet al., 2012; Dianatet al., 2013; Dianat and Karimi, 2014; AlShayhan and Saadeddin, 2018), in Thailand, the schoolbag load has been found to be less than $10 \%$ of the body weight in this population (Deesungnern and Thawinchai, 2014), which is within the recommendations or guidelines for backpack carrying. Therefore, evidently, there should be other risk factors.

Carrying style should be another risk factor as far as MS pain in relation to schoolbag usage is concerned. In Thailand, the majority of schoolbags are backpacks without waist and chest straps (Tunsura and Karoonsupcharoen, 2009; Deesungnern and Thawinchai, 2014), which means that there are two types of carrying styles, the one-strap carrying style and the two-strap carrying style. A previous study (Pascoe et al., 1997) revealed by using the 3D motion analysis that one-strap backpack carrying with $17 \%$ of BW backpack load caused postural changes including lateral spinal deviation and shoulder elevation. Similarly, the study in preadolescent using camera based analysis with digitizing software reported that carrying a backpack weighing 15\% of body weight change all postural angles (Ramprasad et al., 2010). Another study found that asymmetrical activity between the right and the left parts of the back muscle is clearly observed when heavy shoulder bags are carried on the right-hand side of the body, which might indicate the failure of trunk stabilization and contribute to developing of back pain (Motmans et al., 2006). However, the use of high technology such as the 3D motion analysis based camera, or electromyography, which is costly, is limited in Thailand.

The Reedco's posture score (RPS) (REEDCO, 1974) is a standard posture assessment and graded in the coronal and sagittal views in head-to-foot sequences. The RPS has been extensively used in several populations (O'Neil et al., 1992; Gunther et al., 2005; Booshanam et al., 2011; Roman and Samar, 2015). RPS is simple, easy, and inexpensive to use. For those reasons, RPS should be considered an alternative tool by clinics in Thailand to measure postural changes that take place due to carrying of schoolbags. Therefore, the purpose of this study was to compare postures between carrying no backpacks, carrying backpacks with one strap, and carrying backpacks with two straps in adolescents who had MS pain, using RPS. The results from this study may help to understand postural changes during backpack carrying in terms of use 
of tool assessment and, then, to prevent and/or correct posture, thereby reducing unwanted deformities.

\section{MATERIALS AND METHODS}

\section{Participants}

This study was a single-factor design for repeated measures. The sample size for this study was calculated using program $\mathrm{G}^{*}$ power 3.1.9.2. with F-test (ANOVA: repeated measures, within factors), a power of 0.80 , a 0.05 alpha level, two-tailed, and a 0.25 effect size. The estimated total sample size was 28 . However, this study tended to recruit 30 adolescents aged between 12 years and 15 years from schools in Chiang Mai and nearby. To be included in the study, the participant had to have experienced pain within the neck level to the lumbar level of at least $3 \mathrm{~cm}$ on the visual analog pain scale (from $0 \mathrm{~cm}$ to $10 \mathrm{~cm}$ ), had no any congenital anomalies or scoliosis, and was able to complete all measures. The study was approved by the Institutional Review Boards of the institution (022/2556). A parent or guardian of the participants provided permission for their participation and the participants provided informed assent.

\section{Outcome measures}

Reedco's posture score (REEDCO, 1974) is a standard tool and it is administered by visual inspection of 10 postural traits viewed laterally (sagittal view including neck, upper back, trunk, abdomen, and lower back) or from behind (coronal view including head, shoulders, spine, hips, and ankles). The scores are marked as follows: a value of 0 equals poor posture or severe deviation, a value of 5 equals fair posture or minimal to moderate deviation, and a value of 10 equals good posture or normal alignment. The maximum score of 100 indicates good posture and a score of $59 \%$ or less is recorded as postural dysfunction (O'Neil et al., 1992). There was good inter-rater reliability (alpha coefficient $=0.899-0.915$ ) (Healthcare Therapy Services, 2009). In addition, before using the score, this study had found the test-retest reliability of this test to be good (ICC $=0.81-0.95)$.

\section{Procedure}

Before evaluating the posture, the participants were asked to complete personal data form including the participants' characteristics and backpack information. Then the participants' height and weight were measured. Next, in order to assess the posture using RPS, the female participants were asked to wear a bodysuit, while the male participants were asked to take off their shirts 
(Figure 1). Because the backpack would hide the body alignment during posture assessing, markers were attached overlying the anatomical landmarks on the $7^{\text {th }}$ cervical spine, bilateral acromion process, $5^{\text {th }}$ lumbar spine, and bilateral iliac case, as shown in Figure 1. After that, the participants were made to stand before the postural grid at the provided spot with shoes off in three different directions: front (coronal view), back (coronal view), and the sideway which the participant preferred (sagittal view); and three different conditions: carrying no backpack, carrying a one-strap backpack, and carrying a two-strap backpack. Whether to rest or not between conditions depended on the participant. For each direction and each condition, the participant was photographed. Therefore, nine pictures were taken for each participant. The 'no backpack' condition was performed first and treated as the baseline condition; and then, either carrying the one-strap backpack or carrying the two-strap backpack was randomly carried out by drawing. For carrying the one-strap backpack, the participant could choose his/her preferred side. The backpack load used in this study was the mean of $10 \%$ of the body weights of all the participants, which was approximately $4.78 \pm 0.84 \mathrm{~kg}$ (Table 1). Then, all the pictures were assessed using RPS by an assessor (KF).

\section{Data analysis}

SPSS version 17 was used for analysis of the data. One-way repeated measure ANOVA was used to compare the three different conditions, and the Bonferoni test was used for post hoc analysis with $P<0.05$.
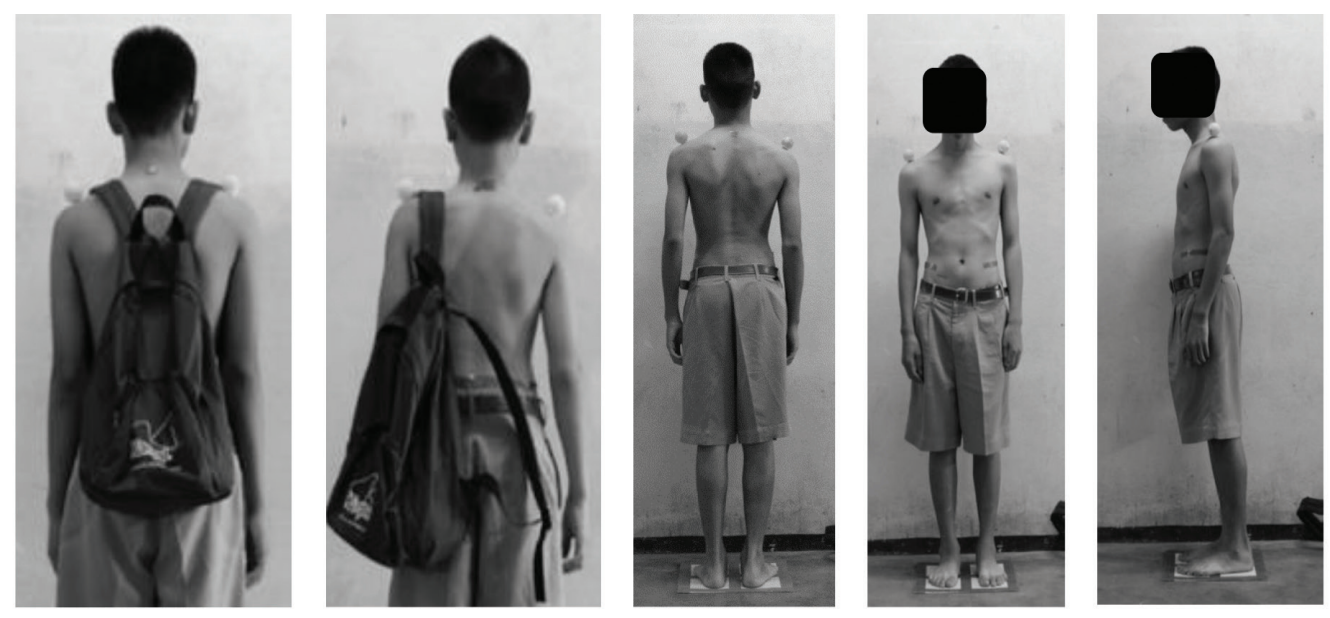

Figure 1. The marker set and the three different views for posture assessment. 
Table 1. Characteristics of participants.

\begin{tabular}{lcc}
\hline \multicolumn{1}{c}{ Characteristic } & Minimum-Maximum & Mean \pm SD \\
\hline Age (years) & $12-15$ & $13.35 \pm 0.85$ \\
Gender (female:male) & $11(42.3 \%): 15$ & $(57.7 \%)$ \\
Height (meter) & $148-175$ & $162.56 \pm 6.56$ \\
Weight (kilogram) & $33-65$ & $47.81 \pm 8.36$ \\
$10 \%$ body weight (kilogram) & $3.30-6.50$ & $4.78 \pm 0.84$ \\
Visual Analog Pain Scale (centimeter) & $3-5$ & $3.35 \pm 0.69$ \\
Baseline Reedco's posture score (\%) & $45-100$ & $83.65 \pm 12.61$ \\
\hline
\end{tabular}

\section{RESULTS}

From 30 adolescents, 4 were excluded because they were outliers. The percent dropout rate from the study was $13.33 \%$, which was acceptable. Among the 26 adolescents, there were 14 adolescents (53.8\%) that carried one-strap backpacks on the right-hand side and 12 adolescents (46.2\%) that carried one-strap backpacks on the left-hand side. The characteristics are shown in Table 1. The adolescents reported pain in the bilateral shoulder region (16 adolescents, 61.5\%), bilateral scapular region ( 2 adolescents, $7.7 \%$ ), lower back region ( 7 adolescents, $26.9 \%$, and left shoulder region ( 1 adolescent, $3.8 \%$ ). The mean and standard deviation values of the posture scores of the 'no backpack carrying', the 'two-strap backpack carrying', and the 'one-strap backpack carrying' conditions are presented in Table 2. The result showed that the lowest postural score was the one-strap backpack carrying $(P<0.05)$.

Table 2. Comparison between carrying styles.

\section{Reedco posture score No backpack Two-strap backpack One-strap backpack}

\begin{tabular}{lccc}
\hline Minimum-Maximum (\%) & $45-100$ & $40-85$ & $35-85$ \\
Mean \pm SD (\%) & $83.65 \pm 12.61$ & $70.00 \pm 12.57^{\mathrm{a}}$ & $63.08 \pm 16.31^{\mathrm{a}, \mathrm{b}}$ \\
\hline
\end{tabular}

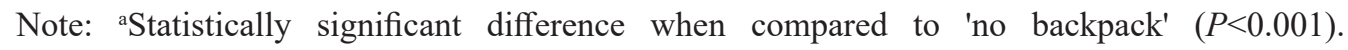

'Statistically significant difference when compared to 'two-strap backpack' $(P=0.005)$. 


\section{DISCUSSION AND CONCLUSION}

The baseline postural score of this current study ranged between $45 \%$ and $100 \%$. The cut-off score indicated that postural dysfunction is less than $59 \%$ (O'Neil et al., 1992). Therefore, the result suggests that some participants already had poor posture. This poor posture might occur in early age. Ruttanaseeha et al. (2009) had reported that $81 \%$ of the primary school students (aged 6 to 9 years old) had carried school bags that weighted exceeding the recommended weight and girls tended to carry heavier bag than boys did. Therefore, there should be an investigation of the poor posture in the primary school students. Specifically, the posture comparison between boys and girls is recommended to be studied in the future.

Decreased scores were found in the sagittal view because of increase in the neck extension or chin protruding out, upper thoracic kyphosis, lumbar lordosis, and/or trunk leaning forward. Although the backpack load was within the recommended limits, that is, $10 \%$ of the body weight, it caused poor posture immediately with two-strap carrying (14.62\% decrease from 'no backpack') and one-strap carrying (20.97\% decrease from 'no backpack'). Similar to previous studies (Pascoe et al., 1997; Chow et al., 2007; Ramprasad et al., 2010), it was observed that at $10 \%$ of body weight, carrying backpacks could cause postural changes not only in the sagittal view but also in the coronal view including one shoulder being higher than the other and/or curving of the spine laterally.

Besides the backpack load, as hypothesized, the carrying style also affects the posture, as found in this study; however, the limitations of the current study were that there were no control group and visual analog scale (VAS) scale during the carrying of the backpack. The one-strap carrying style exhibited the most postural changes. Though this study used RPS with some modification and visual inspection from photos, the findings were similar to those of previous studies that used high technology motion capture (Pascoe et al., 1997; Negrini and Negrini, 2007; Ramprasad et al., 2010). The current study revealed that during one-strap carrying, posture changes were found both in the sagittal plane including head alignment, shoulder level, and pelvic level, and in the coronal plane including upper trunk kyphosis and lower lumbar lordosis. Previous studies reported shoulder elevation and lateral spinal deviation from the weight when the backpack was carried over one shoulder (Pascoe et al., 1997; Negrini and Negrini, 2007). Therefore, evidently, these results indicate that one-strap carrying causes asymmetry of the body in both mediolateral and anterioposterior directions. Contrarily, in two-strap carrying, the body needs to adjust the posture in the anterioposterior direction because the load during two-strap carrying is posteriorly (Motmans et al., 2006; Ramprasad et al., 2010) and is distributed to 
the sides of the body evenly. Therefore, the body is able to keep its balance in order to maintain the center of gravity which is the base of support (Negrini and Negrini, 2007).

In conclusion, it is not only load that causes bad posture but also the carrying style. Therefore, information regarding carrying style should be included in the prevention and intervention program. In addition, to early detect musculoskeletal pain in adolescences, the RPS can be recommended for use in clinics in Thailand as an alternative to or in combination of the questionnaire. However, it needs to be modified for proper evaluation of posture during backpack carrying.

\section{ACKNOWLEDGEMENT}

The authors would like to thank the participants and their parents for participating in this study.

\section{REFERENCES}

AlShayhan, F.A., and Saadeddin, M. 2018. Prevalence of low back pain among health sciences students. European Journal of Orthopaedic Surgery \& Traumatology. 28: 165-170. https://doi.org/10.1007/s00590-017-2034-5 Booshanam, D.S., Cherian, B., Joseph, C.P., Mathew, J., and Thomas, R. 2011. Evaluation of posture and pain in persons with benign joint hypermobility syndrome. Rheumatology International. 31: 1561-1565. https://doi.org/10.1007/s00296-010-1514-2

Chatchawan, U., Puntumetakul, R., Peungsuwan, P., Wanpen, S., Boonprakob, Y., Phadungkit, S., and Puntumetakul, M. 2013. Prevalence of musculoskeletal symptoms in Khon Kaen University students, Thailand. Journal of Medical Technology and Physical Therapy. 25: 193-202.

Chiang, H.Y., Jacobs, K., and Orsmond, G. 2006. Gender-age environmental associates of middle school students' low back pain. Work. 26:19-28.

Chow, D.H., Leung, K.T., and Holmes, A.D. 2007. Changes in spinal curvature and proprioception of schoolboys carrying different weights of backpack. Ergonomics. 50: 2148-2156. https://doi.org/10.1080/0014013 0701459832

Deesungnern, S., and Thawinchai, N. 2014. A survey of factors associated with schoolbag carrying and pain during schoolbag usage in high school students of Darawittayalai School, Chiang Mai. Thai Journal of Physical Therapy. 36: 70-78. 
Dianat, I., Javadivala, Z., Asghari-Jafarabadi, M., Asl Hashemi, A., and Haslegrave, C.M. 2013. The use of schoolbags and musculoskeletal symptoms among primary school children: are the recommended weight limits adequate? Ergonomics. 56: 79-89. https://doi.org/10.1080/ 00140139.2012.729612

Dianat, I., and Karimi, M.A. 2014. Association of parental awareness of using schoolbags with musculoskeletal symptoms and carrying habits of schoolchildren. Journal of School Nursing. 30: 440-447. https:// doi.org/10.1177/1059840513509110

Gunther, J., Kolodziej, T., Bish, C., and Tiberi, J. 2005. Test-retest and interrater reliability of two postural measures in post-menopausal women with osteoporosis. Journal of Geriatric Physical Therapy. 28: 122.

Healthcare Therapy Services. 2009. Reedco posture score sheet. Retrieved from https://goo.gl/ZXEwXz

Moore, M.J., White, G.L., and Moore, D.L. 2007. Association of relative backpack weight with reported pain, pain sites, medical utilization, and lost school time in children and adolescents. Journal of School Health. 77, 232-239. https://doi.org/10.1111/j.1746-1561.2007.00198.x

Motmans, R.R., Tomlow, S., and Vissers, D. 2006. Trunk muscle activity in different modes of carrying schoolbags. Ergonomics. 49: 127-138. https://doi.org/10.1080/00140130500435066

Negrini, S., and Negrini, A. 2007. Postural effects of symmetrical and asymmetrical loads on the spines of schoolchildren. Scoliosis. 2: 8 . https://doi.org/10.1186/1748-7161-2-8

O’Neil, M.B.,Woodard, M., Sosa, V., Hunter, L., Mulrow, C.D., Gerety, M.B., and Tuley, M. 1992. Physical therapy assessment and treatment protocol for nursing home residents. Physical Therapy. 72: 596-604.

Pascoe, D.D., Pascoe, D.E., Wang, Y.T., Shim, D.M., and Kim, C.K. 1997. Influence of carrying book bags on gait cycle and posture of youths. Ergonomics. 40: 631-641. https://doi.org/10.1080/001401397187928

Ramprasad, M., Alias, J., and Raghuveer, A.K. 2010. Effect of backpack weight on postural angles in preadolescent children. Indian Pediatrics. 47: 575-580.

REEDCO. 1974. Reedco's posture score. Auburn, NY.

Roman, G.A., and Samar, V. 2015. Workstation ergonomics improves posture and reduces musculoskeletal pain in video interpreters. Journal of Interpretation. 24: 7. 
Ruttanaseeha, W., Sahunin, A., Supad, P., Chuasathuchon, P., Thanetphonkul, R., Techabunyarat, R., Parima, E., Kessomboon, P., and Sinsupan, N. 2009. Study of weight excess of year 1-4 students' school bags at Khon Kaen University Primary Demonstration School. Srinagarind Medical Journal. 24: 2-8.

Sharan, D., Ajeesh, P.S., Jose, J.A., Debnath, S., and Manjula, M. 2012. Back pack injuries in Indian school children: risk factors and clinical presentations. Work. 41(Suppl 1): 929-932. https://doi.org/10.3233/WOR-2012-0265929

Sihawong, R., Chansirinukor, W., and Akamanon, C. 2006. Prevalence of low back pain among high school students in Bangkoknoi district. Thai Journal of Physical Therapy. 28: 35-42.

Tunsura, W., and Karoonsupcharoen, O. 2009. Effect of backpack weight on postural change and pulmonary function in schoolgirls. Thai Journal of Physical Therapy. 32: 13-20. 